\title{
Patient Development at a Glance: An Evaluation of a Medical Data Visualization
}

\author{
Margit Pohl ${ }^{1}$, Sylvia Wiltner ${ }^{1}$, Alexander Rind ${ }^{2,3}$, Wolfgang Aigner ${ }^{2,3}$, \\ Silvia Miksch ${ }^{2,3}$, Thomas Turic ${ }^{3}$, and Felix Drexler ${ }^{4}$ \\ ${ }^{1}$ Research Group Human Computer Interaction, \\ Vienna University of Technology \\ margit@igw.tuwien.ac.at, s.wiltner@aon.at \\ ${ }^{2}$ Institute of Software Technology \& Interactive Systems, \\ Vienna University of Technology \\ \{rind, aigner, miksch\}@ifs.tuwien.ac.at \\ ${ }^{3}$ Department of Information and Knowledge Engineering (ike), \\ Danube University Krems \\ ${ }^{4}$ Landesklinikum Krems, Austria
}

\begin{abstract}
This paper describes the results of an evaluation study of a prototype for the visualization of time-oriented medical data. Subjects were nine physicians. The prototype combines well-known visual representation techniques and extensive interaction techniques. The aim of the study was to assess the system's usability and whether the prototype solved relevant problems of physicians in hospitals. It was found that one of the great advantages of the system was that it allowed physicians to see the development of the patients at one glance. It was also shown that users clearly preferred an easy to learn and understand design and familiar visualizations.
\end{abstract}

Keywords: interaction techniques, user study, time-orientated data, visual exploration, medical data.

\section{Introduction}

Physicians treating patients with chronic diseases usually have to deal with large amounts of time-oriented data. It is often difficult to get an overview of this data. Information Visualization (InfoVis) might make it easier to derive relevant insights from this data and get a quick idea of what factors influence the patients' development over time. During the project described in this paper, a prototype of an InfoVis system to solve this problem was developed. This prototype is highly interactive to allow the physicians to accommodate it to their needs and to provide them with different views on the data. It was evaluated with nine physicians. The goal of this evaluation was to find out whether the prototype solved the needs of the physicians and whether the visualizations and interactive features of the systems were easy to learn and useful. The results of this work are not only relevant for the system at hand but also for similar systems that represent large amounts of time-oriented medical data using interactive features and a linear time axis as a metaphor. 


\section{Related Work}

Computers play an increasing role in health care, e.g. in electronic medical records. Sainfort et al. [12] report that possible advantages of electronic medical records include better access and availability of patient information for physicians and the availability of full-text search. Patel et al. [6] point out that electronic medical record systems might lead to a lack of time-oriented information concerning the development of patients and that novel features of such systems might be used to overcome this problem. Patel et al. [5] describe thinking and reasoning in medical diagnosis in great detail. They argue that these processes are influenced by the specific affordances of computer-based systems. The design of such systems is, therefore, of great importance.

Making medical records visually accessible is a prominent research area. Therefore, we can only present few, most relevant contributions here and refer the readers to a more extensive review [1].

The Graphical Summary of Patient Status (GSPS) [8] and LifeLines [7] are seminal works in this area. While GSPS and Clinical Care Patient Data Visualization (CPDV) [4] show numerical variables in scatter plots, LifeLines displays nominal variables in a similar fashion to our timeline chart. Midgaard [2] extends LifeLines with a semantic zoom chart technique that shows more details as the chart is enlarged. These systems offer varying degrees of flexibility. Most of them, with the exception of Midgaard, rely on a single visualization technique for either numerical or nominal variables. Further, most of them, with the exception of CPDV, do not allow the physician to rearrange, add, or remove variables from the display.

\section{Analysis of Medical Requirements}

For a thorough analysis of users, tasks, and data we involved physicians in a usercentered design process from the very beginning, as described in [11]. We developed our prototype based on the following requirements, which we elicited from introductory interviews of five physicians and contextual observations: (1) Simple user interface and visualizations, which are particularly clear and allow for unambiguous insights; (2) flexibility to work with various medical variables and combine different groups of variables; (3) time-oriented data representing patient development over time; (4) multiple patients; and (5) interactivity through a variety of interaction techniques [11]. Furthermore, we had repeated workshops during the development phase to discuss and refine the design based on feedback of InfoVis experts, usability experts, and physicians.

In order to provide a realistic medical scenario for the design process and the user study, we worked with data and tasks from a diabetes outpatient clinic. The data is composed of medical test results and therapy prescriptions that are collected at checkup examinations scheduled about every two months. Though, the examination intervals and the provided data items may vary largely depending on the patient's condition. Physicians are interested in whether the condition of a diabetes patient improves or worsens. How the improvement of some variables relates to the development of others is of particular importance. They also need to know which types of therapy a patient already had and how these affected medical tests. 


\section{Design of the VisuExplore System}

Next, we describe how we combined simple and well-known visual representation techniques with extensive interaction techniques that meet four of the five medical requirements presented above. Visually exploring the developments and relationships of multiple patients imposes a different set of tasks to support. Therefore, a separate system for multiple patients has been developed and is reported in [10].

VisuExplore (Fig. 1) displays medical variables in multiple diagrams, which are aligned along a horizontal time axis. Thus, it encodes time by positioning on a common scale, which is perceptually effective [3] and should be easy to learn. The visual representation techniques used in the diagrams are well known and quite simple. To allow for flexibility VisuExplore provides several representation techniques: Line plots and bar charts for numerical variables and event charts and timeline charts for nominal variables. A diagram may also display two or more variables. Furthermore, we extended the system with five advanced representation techniques: a semantic zoom chart (cp. [2]), a step chart, a silhouette graph, a horizon graph (cp. [9]), and a document browser. Though, in the user study (Sect. 5) we only evaluated simple techniques because the introductory requirements analysis called explicitly for simple visualizations.

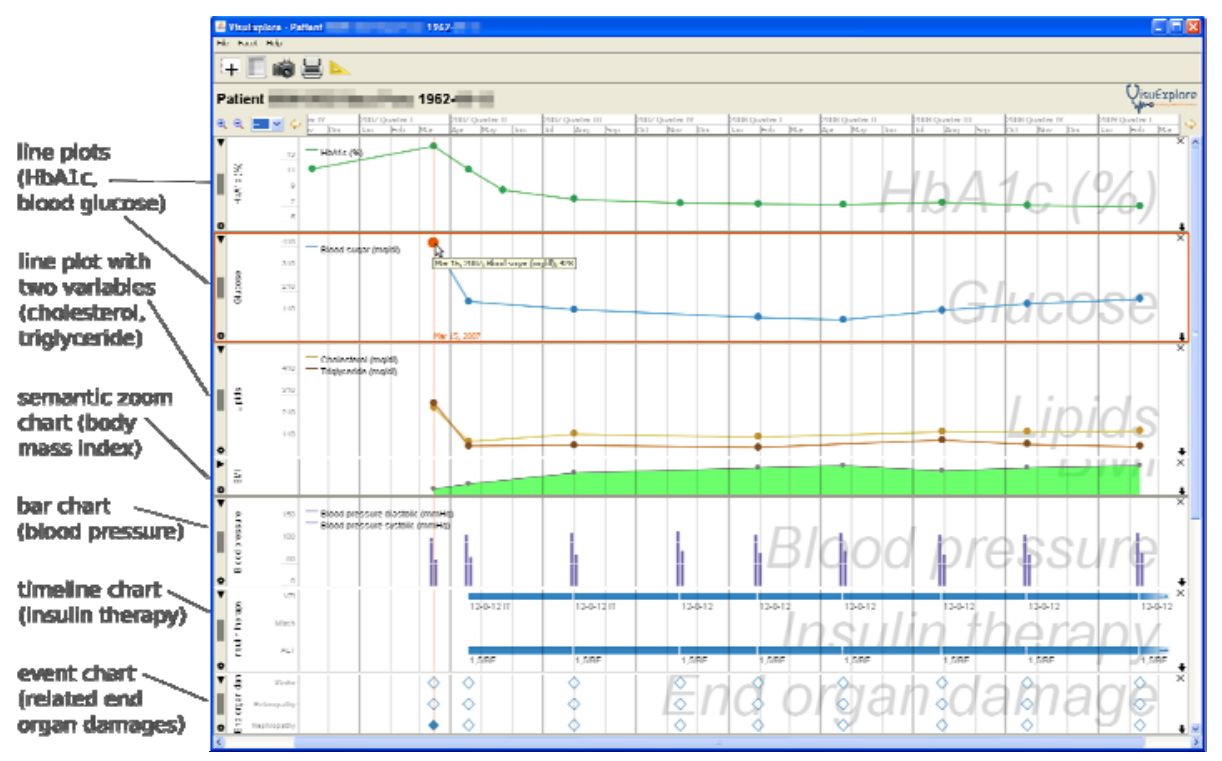

Fig. 1. Exploring a medical record using VisuExplore

VisuExplore starts with a predefined set of diagrams, which is customizable to accommodate for different medical specialties. In our diabetes scenario we provided a line plot of blood glucose, a second line plot of cholesterol and triglyceride, a timeline chart of insulin therapy, etc. But this set is just an initial aid for physicians, as VisuExplore offers a number of interaction techniques to adapt the diagrams. 
Physicians can create new diagrams and close existing ones. They can drag diagrams to change their order, and thus compare developments in two diagrams more easily. They can toggle each diagram between a large and a collapsed view or drag its border to change the height continuously. Thereby our representation techniques adapt to their available screen space and show more or less detail. Each diagram has a control panel for its settings. For example, a line plot setting specifies whether the y-axis zooms to the values of the current patient or extends to a domain-specific value range.

If users are interested in a specific time interval, they can zoom in continuously with the mouse or select from a predefined set of meaningful zoom steps such as "1 year" or "3 months". They can scroll horizontally across time as well as vertically along diagrams.

Further interaction techniques of VisuExplore support physicians in the exploration of the medical data. As they move the mouse across the window, a vertical line follows the cursor to help them find co-occurring items. Using tool tips and a table panel they can read exact data values. The table panel shows all items of a diagram textually and can be sorted by any column (e.g. variable value). Physicians can select one or more data items either in the diagram or in the table panel, whereupon selections are linked. Finally, VisuExplore provides a tool for physicians to measure the duration of a time interval or the time difference between two items, especially to judge a possible cause-effect relationship. With the measure tool, they can drag a metaphorical tape measure from a reference point to the current mouse location, which works as well across multiple diagrams (Fig. 2). Similar tools are often found in geospatial visualizations, but are uncommon for time-oriented data.

\section{Description of the Investigation}

The aim of the user study was, on the one hand, to assess the usability of the system and, on the other hand, to check whether the system aids in solving relevant tasks of medical doctors in hospitals. The most relevant research questions were:

1. Which of the physicians' tasks can be supported by the proposed system?

2. In which departments of the hospital should the system be used?

3. Which forms of interaction are most useful?

4. Which of the proposed visualizations for the variables are appropriate?

The subjects were nine physicians from two hospitals. Seven of them were women, two men. This reflects the fact that many of the physicians in the two hospitals taking part in this study are women. Most of them were under 40 years of age, only one was between 50 and 60 . Four of them were specialists in diabetes care, the others were familiar with diabetes. We selected experts in fields where the tool might realistically be applied, especially in fields with large amounts of time-dependent data (e.g. in the treatment of pulmonary diseases). The subjects were not only early adopters but had various degrees of previous knowledge of computer usage.

It is well known that it is difficult to do user testing with professionals, especially because of their time constraints. Therefore, we tried to organize the investigation in such a way to get the most out of the limited time the physicians were available. First, 
we gave them a short introduction into the system $(10 \mathrm{~min})$, then they had to solve three tasks. An example for such a task is: "Please look at the data of three patients $(\mathrm{A}, \mathrm{B}, \mathrm{C})$, especially at blood sugar, cholesterol and body mass index. Can you see any relationships between the three parameters for these three patients?" These tasks were developed together with a diabetes expert from the hospital. The time subjects spent working on the tasks was approximately 20 minutes. In the end, we conducted a short qualitative interview with open-ended questions (planned were 10 minutes, but some interviews lasted for 30 minutes). The interview partners sometimes indicated relevant features on the screen and gave us concrete examples to substantiate their views.

The main method used in this study to answer the research questions was interviews. The results presented in this paper are based on these interviews. The questions asked were basically refined versions of the research questions outlined above. We asked them specifically to comment on the advantages and disadvantages of all available interaction methods. The interviews were then transcribed. The interpretation of such interviews is often difficult because interview partners utter conflicting statements (e.g. they do not appreciate a certain feature, but would use it if improved in well-defined ways). Therefore, quantitative scores often do not reflect such results. In the study, only the simple representation and interaction techniques were tested.

\section{Results}

In this section, we give an overview of the results of the interviews with the physicians.

\subsection{Advantages of the System (Research Question 1)}

A great advantage of the system mentioned by all participants in the study is the fact that users can see the development of the patients at one glance. One very important input mentioned by the physicians in the requirements analysis was that for the treatment of patients with chronic diseases they have to read many reports on diagnostic findings. This may take up much of their time, and it is a great challenge to derive an overall impression of the patients' development from these reports. The VisuExplore system can support physicians considerably in this activity. It apparently solves the physicians' problem with information overload.

In addition, most of the participants mentioned that the system was easy to learn and understand. The visualization methods were found to be intuitive and clear.

\subsection{Potential Areas of Application (Research Question 2)}

Most of the physicians agreed that the VisuExplore system could best be applied for outpatient care of chronic diseases (diabetes, treatment of cardiac insufficiency, cancer, etc.). According to the interviewed physicians, it would not be so useful for inpatient treatment because most of these patients stay in contact with the hospital for 
much shorter periods of time. Another possible application mentioned by some subjects is that the system could be used to communicate to the patients the state of their health. Some physicians thought that the patients might be more impressed by a visual representation of the data than by just showing bare numbers. Furthermore, it was mentioned that it might also be used for teaching purposes.

\subsection{Interaction (Research Question 3)}

Resizing Diagrams (Fig. 2): In the VisuExplore system, it is possible to increase/decrease the height of the diagrams. The physicians' attitude to this possibility was mixed. Three found this quite useful, others thought this was not necessary or even misleading (four noted that when decreasing the height of the diagrams, differences between values are also considerably decreased giving a wrong impression of the patients' development). In contrast, some physicians liked this possibility because in they could get a better overview of all necessary values.

Moving Diagrams: Seven of the physicians explicitly liked this possibility. They commented that they found it interesting to create their own view of the data so that they could use the system most efficiently. Some of them also mentioned that they would create this view once and then stick to it for most cases, but that this view would be different for every physician.

Measure Tool (Fig. 2): Concerning the measure tool, the physicians' reactions were mixed. Only three really liked this feature and others found this unnecessary or confusing. One subject proposed that the tool should calculate the mean value of the data in the selected time interval.

Pan and Zoom: Both were found to be interesting under certain conditions, especially when there were many data items with changing values. However, it must be mentioned that in many cases the line plots in combination with a table showing the concrete values can provide enough information to get a clear overview.

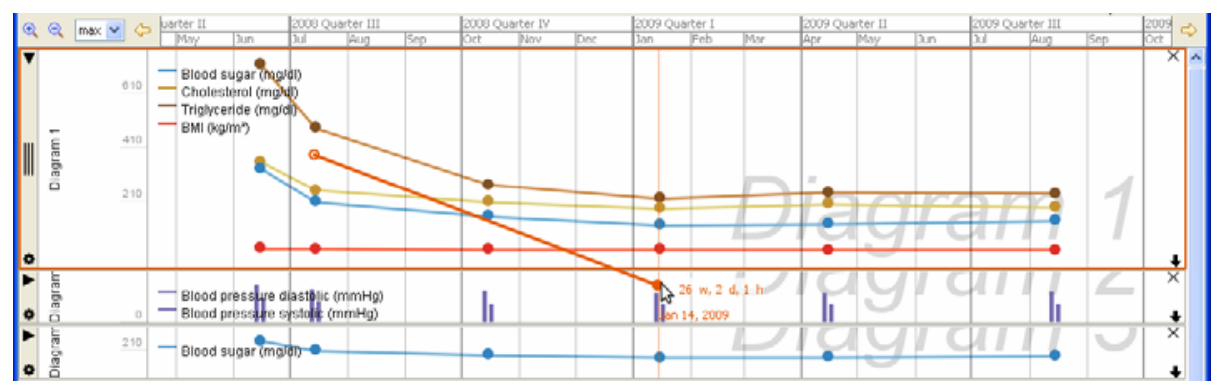

Fig. 2. The first diagram combines several variables. The second and third diagram are resized. The measure tool is shown (slanted orange line) going from the first to the second diagram.

Scrolling: We observed that the activity physicians adopted most was scrolling. This is partly due to the visualization itself (one diagram below another), but we also observed that they seemed to prefer scrolling to closing or resizing diagrams. 


\subsection{Visualizations (Research Question 4)}

Several Variables in one Diagram (Fig. 2): There were also mixed reactions concerning this issue. Four physicians preferred to combine several variables represented by line plots (e.g. cholesterol, triglyceride, BMI, and blood sugar) in one diagram because of the compact form and the possibility to see everything at a glance. Other physicians found this confusing, especially because of the different scales involved. Furthermore, BMI has a different unit of measurement. In addition, they found too many lines in one diagram confusing. There were, apparently, different approaches to solve the given tasks. It should be pointed out, however, that all physicians found plausible solutions for the tasks while using different methods for reaching these solutions.

Methods of Visualization (Fig. 1): The system offered several methods of visualization (line plots, bar charts, etc.). There was a general consensus among physicians that line plots were the best form of representation. They argued that line plots are well known and can therefore be interpreted easily. Line plots can show the development in a convincing manner, outliers can be identified easily. Bar charts for blood pressure are fine, but even here they would have preferred line plots.

In general, well-known visualizations were preferred. Because of their timeconstraints and workload, physicians are very reluctant to familiarize themselves with unknown forms of visualization.

\section{Conclusion and Future Work}

Our study indicates that the system is considered to be useful for supporting the therapy of patients with chronic diseases like diabetes.

During the requirement analysis physicians expressed their wish for a system that is easy to understand and learn. It was often mentioned during the user study that our system satisfies these needs and that it is very simple and intuitive.

Physicians also showed a clear preference for line plots because they were familiar with this visualization and could therefore easily and quickly interpret them. Other forms of visualizations should probably be used sparingly.

There are other features of the system where flexibility seems to be welcome and strong personal preferences of the physicians could be observed (e.g. combining several variables in one line plot, moving and resizing diagrams). It was also stated that personalization of the interface according to individual needs is an issue.

The evaluation study indicates that four of the five requirements described in Sect. 3 were fulfilled by the system. The interface is simple and easy to learn, the system is flexible and can be adapted to the users' needs, time-oriented data can be understood at a glance, and a reasonably level of interactivity is supported. The fifth requirement (comparing multiple patients) is fulfilled by another visualization system called TimeRider, which can be combined with VisuExplore [10].

The investigation described in this paper is part of a larger, ongoing study. The aim of that study is to clarify in more detail what strategies users of information visualizations adopt and to identify interaction patterns so that information visualizations can be designed to support users more efficiently. 
Acknowledgments. This work was supported by the Bridge program of the Austrian Research Promotion Agency (project no. 814316) and conducted in cooperation with Danube University Krems, Vienna University of Technology, NÖ LandesklinikenHolding, Landesklinikum Krems, NÖGUS, systema Human Information Systems.

\section{References}

1. Aigner, W., Kaiser, K., Miksch, S.: Visualization techniques to support authoring, execution, and maintenance of clinical guidelines. In: ten Teije, A., Lucas, P., Miksch, S. (eds.) Computer-based Medical Guidelines and Protocols: A Primer and Current Trends, pp. 140-159. IOS, Amsterdam (2008)

2. Bade, R., Schlechtweg, S., Miksch, S.: Connecting time-oriented data and information to a coherent interactive visualization. In: Proc. ACM SIGCHI Conf. Human Factors in Computing Systems (CHI), pp. 105-112 (2004)

3. Cleveland, W.S., McGill, R.: Graphical Perception: Theory, Experimentation, and Application to the Development of Graphical Methods. J. Am. Statistical Assoc. 79(387), 531-554 (1984)

4. Faiola, A., Hillier, S.: Multivariate relational visualization of complex clinical datasets in a critical care setting: A data visualization interactive prototype. In: Proc. Int. Conf. Information Visualisation (IV), pp. 460-468. IEEE, Los Alamitos (2006)

5. Patel, V.L., Arocha, J.F., Zhang, J.: Thinking and Reasoning in Medicine. In: Holyoak, K.J., Morrison, R.G. (eds.) The Cambridge Handbook of Thinking and Reasoning, pp. 727750. Cambridge University Press, Cambridge (2007)

6. Patel, V.L., Kushniruk, A.W., Yang, S., Yale, J.-F.: Impact of a Computer-based Patient Record System on Data Collection, Knowledge Organization, and Reasoning. J. Am. Med. Inform. Assoc. 7(6), 569-585 (2000)

7. Plaisant, C., Mushlin, R., Snyder, A., Li, J., Heller, D., Shneiderman, B.: LifeLines: using visualization to enhance navigation and analysis of patient records. In: Proc. AMIA Symp., pp. 76-80 (1998)

8. Powsner, S.M., Tufte, E.R.: Graphical summary of patient status. Lancet 344(8919), 386389 (1994)

9. Reijner, H.: The development of the horizon graph. In: Proc. Vis. 2008 Workshop From Theory to Practice: Design (2008)

10. Rind, A., Aigner, W., Miksch, S., Wiltner, S., Pohl, M., Drexler, F., Neubauer, B., Suchy, N.: Visually exploring multivariate trends in patient cohorts using animated scatter plots. In: Proc. Int. Conf. Human-Computer Interaction, HCI-I (forthcoming, 2011)

11. Rind, A., Miksch, S., Aigner, W., Turic, T., Pohl, M.: VisuExplore: gaining new medical insights from visual exploration. In: Hayes, G.R., Tan, D.S. (eds.) Proc. Int. Workshop on Interactive Systems in Healthcare (WISH@CHI2010), pp. 149-152. SIGCHI (2010)

12. Sainfort, F., Jacko, J.A., Booske, B.C.: Human-Computer Interaction in Health Care. In: Jacko, J.A., Sears, A. (eds.) The Human-Computer Interaction Handbook, pp. 808-822. Lawrence Erlbaum Associates, Mahwah (2003) 